\title{
Automatic segmentation of the sphenoid sinus in CT-scans volume with DeepMedics 3D CNN architecture
}

\author{
Type of article: Original \\ Kamal Souadih ${ }^{1}$, Ahror Belaid ${ }^{1}$, Douraied Ben Salem² \\ ${ }^{1}$ Medical Computing Laboratory (LIMED), University of Abderrahmane \\ Mira, 06000, Bejaia, Algeria \\ 2 INSERM UMR 1101, Laboratory of Medical Information Processing (LaTIM), \\ 5 avenue Foch, 29200 Brest, France, \\ Neuroradiology and Forensic Imaging Department, \\ CHRU Brest, La Cavale Blanche Hospital. Boulevard Tanguy Prigent, 29609 Brest, \\ France.
}

\begin{abstract}
.
Today, researchers are increasingly using manual, semi-automatic, and automatic segmentation techniques to delimit or extract organs from medical images. Deep learning algorithms are increasingly being used in the area of medical imaging analysis. In comparison to traditional methods, these algorithms are more efficient to obtain compact information, which considerably enhances the quality of medical image analysis system. In this paper, we present a new method to fully automatic segmentation of the sphenoid sinus using a $3 \mathrm{D}$ (convolutional neural network). The scarcity of medical data initially forced us through this study to use a 3D CNN model learned on a small data set. To make our method fully automatic, preprocessing and post processing are automated with extraction techniques and mathematical morphologies. The proposed tool is compared to a semi-automatic method and manual deductions performed by a specialist. Preliminary results from $\mathrm{CT}$ volumes appear very promising.
\end{abstract}

Keywords: Deep Learning, Biomedical Engineering, 3D Imaging, 3D CNN, CT Scan, Sphenoid Sinus, Automatic Segmentation.

Corresponding author: Kamal Souadih, Medical Computing Laboratory (LIMED), University of Abderrahmane Mira, Bejaia, Algeria. Email: skamelmail@gmail.com

Received: 23 February, 2018, Accepted: 08 Mars, 2019, English editing: 25 Mars, 2019,Published: 01 April, 2019.

Screened by iThenticate..C2017-2019 KNOWLEDGE KINGDOM PUBLISHING.

\section{Introduction}

The sinuses anatomy are complex and very variable [1]. The sphenoid sinus cavity is the most variable from person to others. It is an essential landmark in surgery but it is hard to isolate [2-3-4]. Fig. 1 shows a diagrammatic representation of the sinuses location. Another difficulty is that the sinuses can also to divide into multiple niches that communicate the one with the other through an incomplete bone wall, which further complicates their location [5]. Complexities of spheroidal sinus surgery are easy to avoid if we understand its anatomical features [6]. Sphenoid bone has a deep anatomical location in the skull making it difficult to approach. This deep location may be advantageous in the case of forensic identification. Unlike other sinuses, the sphenoid sinus is well preserved from traumatic damage of external causes. Sphenoid sinuses can be classified into four types [7]:

Conchal: complete missing or minimal sphenoid sinus; 
Pre-sellar: the posterior wall of sphenoid sinus is in front of the anterior wall of the sella turcica

Sellar: the posterior wall of the sphenoid sinus is between the anterior and posterior walls of sella turcica

Post-sellar: the posterior wall of sphenoid sinus is behind the posterior wall of the sella turcica

This classification is based on basic aspects (height, width and depth) and can be used to predict the potential for accidental injury, but they are also useful for individual identification as can be seen in [8].

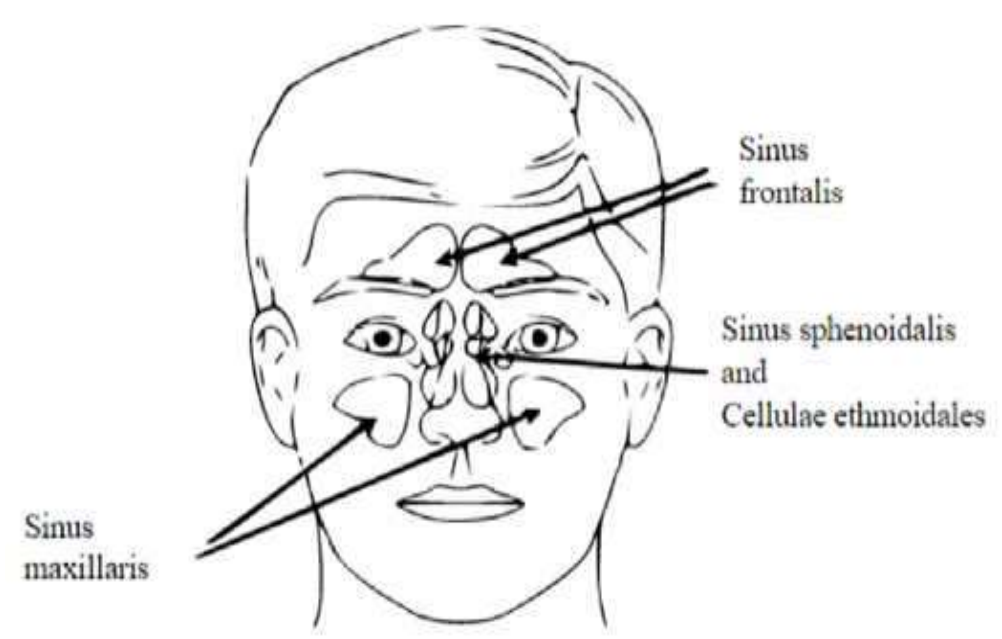

Fig. 1. Diagrammatic representation of paranasal sinuses.

CT scans images are an excellent choice for assessing the anatomy of the sinus because they provide an accurate craniofacial assessment of the bones and the extent of their pneumaization [9-4]. By segmenting 3D CT-images of the sphenoid sinus, we can make useful measurements of its volume anatomy $[10,19,20]$. Image volume segmentation is a technique of marking each voxel in an image and assigning it to a group of voxels defining an anatomical structure. This technique has wide and varied applications in medical research and computer-assisted diagnosis. It makes it possible to extract and recognize organs. It is used too to improve the visualization and permit quantitative measurements. Segmentation is essential too for the construction of anatomical atlases, the search for organ structure shapes and monitoring their evolution through the age [11]. Artificial Intelligence techniques relying on machine learning, more and more, are used for the analysis and segmentation of medical images. In the last years, the appearance of deep learning techniques has contributed significantly improving 
medical image analysis, in which convolutional neural networks (CNNs) are used and that give the ability to learn significant patterns automatically and extract real structures from images $[3,12]$. One of the keys to the success of the $\mathrm{CNN}$ is that it is possible to use the preformed models directly to perform tasks other than those initially planned. It is now easy to download a learned model and then adjust it slightly to adapt it to the application in question [13]. In this work, we are proposing a new method for the automatic segmentation of the sphenoid sinus represented on CT scan volumes using a 3D CNN architecture. The proposed method is robust, fast, and efficient.

\section{Material and method}

Our automatic sphenoid sinus segmentation method consists of three main steps, where the result of the step is the input of another one. The first step is a preprocessing step; we create and transform automatically the images volume given from a PACS to an image of the region of interest. Then, we perform a segmentation with 3D Deep CNN [14] that we adapted and parameterized to produce highly accurate sinus segmentation. Finally, postprocessing based on mathematical morphology operations to perform a sinus measurement and refine a segmentation (Figure.1). This splitting in stages allowed us to improve and simplify the use of $\mathrm{CNN}$ at the CPU level. In the following we describe the method stages:

\subsection{The automatic ROI extraction for the CT-image}

The preprocessing step uses some interesting techniques with slight transformations that are adapted to improve the effectiveness of the specific type of segmentation method used in the next step. These transformations are made so those common parameters can be used for all images of all intensity ranges. In other words, we aim to operate only on a reduced $3 \mathrm{D}$ region, a region of interest centered on the sinus at issue and not on the whole image. This region of interest must be the same in terms of dimensions for all images in the data set of training or test. To achieve this, first a target image with a well-oriented head and a clear sinus was chosen. We manually traced a large rectangle, enough to contain the sinus whatever its shape, size does not exceed $200 \times 200 \times 200$ pixels. This rectangle will also serve as a reference bounding-box. Then, all other database images are registered onto this target image with its bounding box. As the images are coming from different persons, we choose to use a rigid registration, allowing correction of the different positions and orientations arising from the clinical exam. Since the natural size of the skulls is different from one person to another, we have avoided using affine registration [15], which risks distorting the estimation volume that will be used later as a parameter for identification. Thereby, we were able to build a new database consisting only of regions of interest, with the same size as the reference box. 


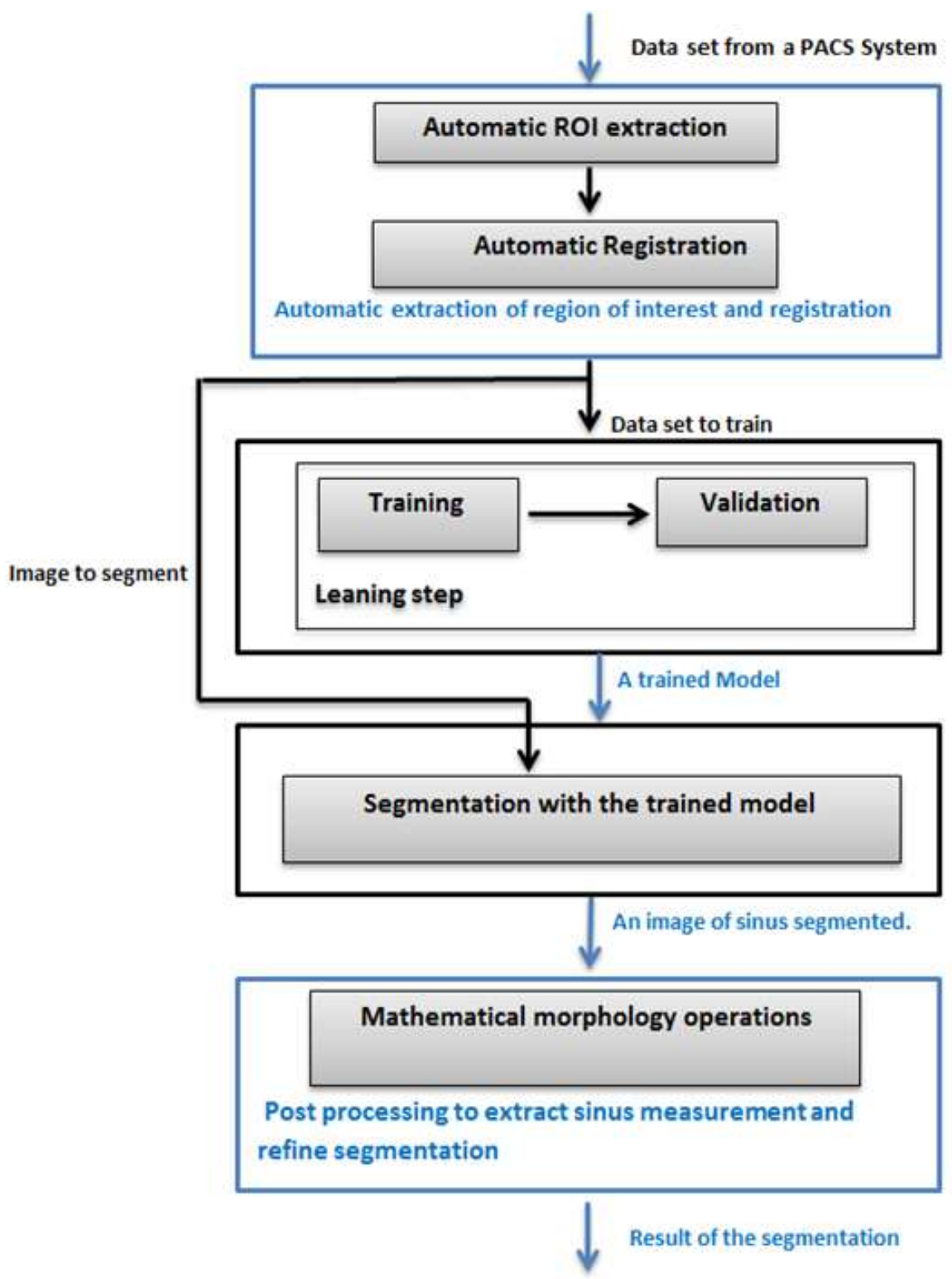

Fig. 2. Flow chart of the sphenoid sinus segmentation scheme.

\subsection{Sinus Segmentation with Deep 3D CNNs}

This step employs the Deep Medic [16] architecture realized as open source architecture for medical images analysis [17], it is an algorithm with an adjustable number of deep layers, double-pathway and 3D CNN, created to segment the volume images brain lesions [14]. This architecture segments MRI 3D images corresponding to a multi-modal 3D patch at multiple scales. In our study we have used the lightweight version CPU-based of this software to drive our sinus automatic segmentation model; in our case, we use one modality and a CT images format. This CPU model gives a satisfactory solution to our problem. The reliability of this algorithm was evaluated when fewer training data were available or filters were used, and this architecture was referenced on the BRATS 2016 Challenge, 
where it performed exceptionally well despite the simplicity of the pipeline [17]. It was demonstrated that it is possible to train this $3 \mathrm{D} C N N$ on a small dataset of 28 volume CT scan images. This network delivered a good result on the task of segmenting ischemic stroke lesions, accomplishing a mean Dice of $64 \%$ (and $66 \%$ after post-processing) on the ISLES 2015 training dataset, ranking among the top entries $\lceil 14\rceil$. This architecture $\lceil 16\rceil$ is based on:

- Two paralleled convolution paths that process inputs at multiple scales to provide a large receiver field for final classification while limiting calculation costs.

-A small convolutional kernel. That gives efficiency to construct CNNs at depth without significantly increasing the number of parameters that can be driven and inspired by the Very deep convolutional networks (VGG) 「181. Designed efficient and productive 3D CNNs thanks to the much smaller calculation required for convolution with small $3{ }^{3}$ kernels.

-A complete convolutional method on image segments in the formation and test phase.

The main algorithm steps, which make up this architecture, are presented in this section. The DeepMedic theoretical background is detailed very clearly in [14]. A summary of each step, which makes up this algorithm follows:

1- Each layer $l \in[1, L]$ consists of $C_{l}$ Feature Maps (FM) also referred to as Channels.

2- Every FM represents a group of neurons that detect a particular pattern (a feature, in the channels of the previous layer).

3- A pattern is defined by kernel weights associated with the FM

4- If the neurons of the $m_{t h} F M$ in the $l_{\text {th }}$ layer are arranged in a $3 D$ grid, their activations constitute the image defined by

$$
y_{l}^{m}=f\left(\sum_{n=1}^{c_{l-1}} k_{l}^{m \cdot n}\right) * y_{l-1}^{n}+b_{l}^{m}
$$

where

$y_{l}{ }^{m}$ is the result of convolving each of the previous layer channels with a $3 D$ kernel, $k_{l}^{m . n}$ is a kernel adding a learned bias $b_{l}{ }^{m}$ applying to a non-linear function $f$, $y_{0}{ }^{n}$ is the input to the first layer, corresponding to the channels of the original input image.

5- Each kernel is a matrix of learned hidden weights $W_{i}^{m, n}$.

6- Each class of segments has a number $C_{l}$.

7- The activations of $C_{l}$ are fed into a position-wise softmax function that produces the predicted posterior

$$
p_{c}=\frac{\exp \left[y_{L}^{c}\right](X)}{\sum_{c=1}^{C_{L}} \exp \left[y_{L}^{c}\right]}
$$


with $y_{L}{ }^{c}$ is the activation of the FM at position $l G N^{3}$

8- The size of the neighborhood of voxels $\phi_{l}$ in the input that influences the activation of a neuron is a receptive field, increases at each subsequent layer and is given by the $3 D$ vector:

$\phi_{l}^{\{x, y, z\}}=\phi_{l-1}^{\{x, y, z\}}+\left(k_{l}^{\{x, y, z\}}-1\right) \tau_{l}^{\{x, y, z\}}$,

where

— $k_{l}$, and $\tau_{l} \in N^{3}$ are vectors expressing the size of the kernels and stride of the receptive field at layer $l$;

$-\tau_{l}=(1,1,1$ is given by the product of the strides of kernels in layers preceding in this system;

$-\phi_{C N N}=\phi_{L}$ is the CNN receptive field; where the receptive field of a neuron in the classification layer corresponds to the image patch that influences the prediction for its central voxel.

9- The dimensions of the FMs in layer l is given by

$$
\delta_{l}^{\{x, y, z\}}=\frac{\left(\delta_{i n}^{\{x, y, z\}}-\phi_{l}^{\{x, y, z\}}\right)}{\tau_{l}^{\{x, y, z\}}}+1 .
$$

10-If an input of size $\delta_{\text {in }}$ is provided, $\delta_{i n}=\phi_{C N N}$ is a size of input patch in the common patch-wise. The FMs of this classification layer have $1^{3}$.

11-CNNs are trained patch-by-patch, and random patches of size $\phi_{C N N}$ are extracted from the training images.

12-To maximize the log-likelihood of the data or, equally, minimize the cross-entropy via the cost function

$J_{D}\left(\theta ; I^{i} ; C^{i}\right)=-\frac{1}{B} \sum_{i=1}^{B} \log \left(P\left(Y=c^{i} \mid I^{i}, \theta\right)\right)=-\frac{1}{B} \sum_{i=1}^{B} \log \left(p_{c}\right)$

where

$B$ is the size of batch, which is then processed by the network for one training iteration of Stochastic Gradient Descent (SGD);

The pair $\left(I^{i}, C^{i}\right), \forall i \in[1, B]$ is the $i_{\text {th }}$ patch in the batch and the true label of its central voxel;

The scalar $p_{C}{ }^{i}$ is the predicted posterior for Class $C^{i}$; and

Regularization terms were omitted for simplicity. Multiple $C^{i}$ Sequential optimization steps over different batches gradually lead to convergence.

13-The classification layer is the activation of the last layer of CNN.

14-Memory requirements and computing times increase with the batch size, which is the limitation of $3 D C N N s$, DeepMedic uses a strategy that exploits the dense inference technique on image segments. Following from Eq.(2), if an image segment of size greater than $\phi_{C N N}$ is given as input to the network, the output is a posterior probability for multiple voxels $V=Q \delta_{l}^{i}$. If the training batches $i=\{x, y, z\}$ are formed of $B$ segments extracted from the training images, the cost function Eq.(3), in the case of densetraining [14] becomes

$J_{D}\left(\theta ; I_{s} ; C_{s}\right)=-\frac{1}{B V} \sum_{s=1}^{B} \sum_{v=1}^{V} p c_{s}^{v}\left(x^{v}\right)$

where $I_{s}$, and $C_{s}$ are the $s$-th segment of the batch and the true labels of its $v_{\text {-th }}$ voxel, $x^{v}$ the corresponding position in the classification FMs and $p_{c} v$ the output of the softmax function. Factor V increases the effective lot size without the corresponding increase in calculation and memory requirements 
DeepMedic architecture is also a deep architecture based on small $3^{3}$ kernels that are faster to convolve with and contain fewer weights [14].

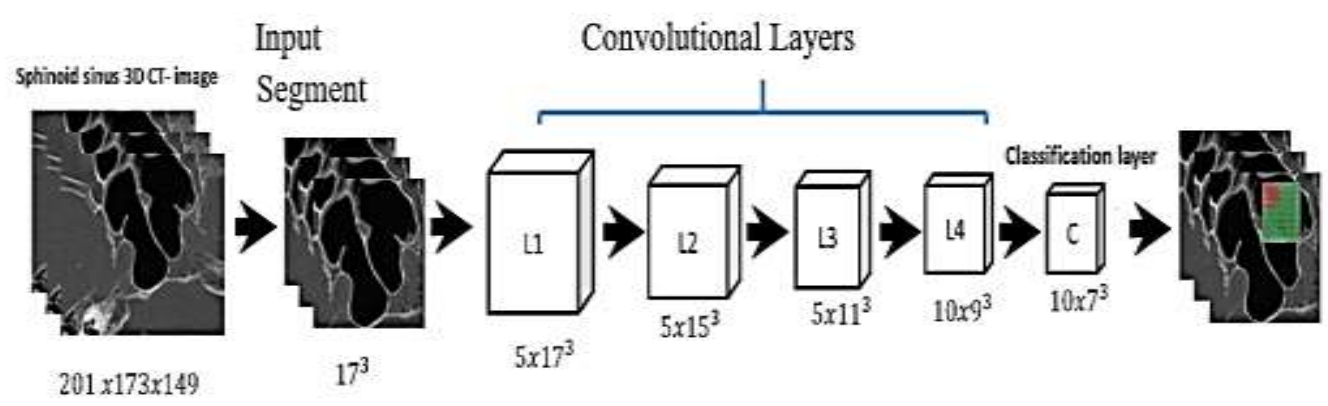

Fig.3. The architecture of the DeepMedic for automatic sphenoid sinus segmentation.

The 3D CNN has been adapted for five layers, with a receptive field of size $17^{3}$ and 1 modality. The classification layer (the last layer) is implemented like a convolutional layer with $1^{3}$ kernels, which enables efficient dense inference. When the network segments an input it predicts multiple voxels simultaneously, one for each shift of its receptive field over the input (see Figure 4). The training time required for convergence of the final system is roughly 20 minutes using a CPU Intel I5-7300 with $2 \times 2.5 \mathrm{GHz}$. Segmentation of a 3D scan of a sphenoid sinus requires 1 minute.

\subsection{Post-processing}

The segmentation result obtained by the 3D CNN of the precedent step method does not make it possible to distinguish between the sphenoid sinus from the other sinuses. The nasal cavities as well as the paranasal sinuses have almost the same gray level intensity. To differentiate the sinuses, we have used a prior knowledge about the positioning of these sinuses. Indeed, the sphenoid sinus is the deepest cavity starting from the front face, and therefore it is the first cavity encountered from the back of the skull at the median. Thus, using the operations of mathematical morphology we have been able to locate the sphenoid sinus. We have first applied an erosion operation to the segmented image, which allows removing the residues, but especially the potential connections between the sphenoid sinus and other cavities. More precisely, erosion operation allows to remove the ostium and to well separate the two hemisinus of the sphenoid sinus. Once the sphenoid sinus cleared, we have subsequently calculated the centers of gravity of all the regions on the image. After sorting the centers coordinates along the coronal axis, the deepest center corresponds, of course, to the region of the sphenoid sinus, or more precisely corresponds to the deepest hemisphere. When the hemisphere is segmented from the rest of the cavities, a dilation operation (with the same parameters as the previews erosion) is applied to recover some details of the shape lost during erosion operation. As can be seen, the detection of the two hemispheres of the sinus is sequential. Indeed, after removing the first 


\section{Result}

\subsection{Dataset}

The data set used includes 24 head CT volumes images, which were taken on a CT scanner with several helical detectors. All CT scans with a disease involving the sphenoid and its surroundings structures, but also with mucosal thickening of the sinuses, mucosal sinus thickening or an anomaly in the content of the sinuses were not included in the study. After the preprocessing step, 3D CT-images less than 200 x 200 x 200 have obtained where 15 images were used to train the algorithm (training and validation) and 9 images to test the training. A manual segmentation of spheroid sinus for each image on train data set was performed manually, so we did this assisted by a radiologist.

\subsection{Results}

An example of 3 segmentations is reported in Figure 4. It shows the result of the segmentation and the extracted sphenoid sinus as explained in the previous sections. The segmentation is performed using the $3 \mathrm{D} \mathrm{CNN}$ and affine with the morphological operations.

Images

Fig.4. Segmentation examples for 3 CT-images, shows a superior, left, interior and front views.

\subsection{Validation}

To evaluate the precision and reliability of our automated approach, we have compared the results of segmentation of the same sphenoidal sinus of our tool with the ITK-SNAP a semi-automatic segmentation and with manual segmentation conducted with an experienced radiologist using a standard procedure. Each image 
segmented by accurately drawing the contours of the sphenoid sinus following the surface of the inner bone in an axial direction. An example of the manual segmentation process of the spheroid sinus of a slice is presented in Figure 5.

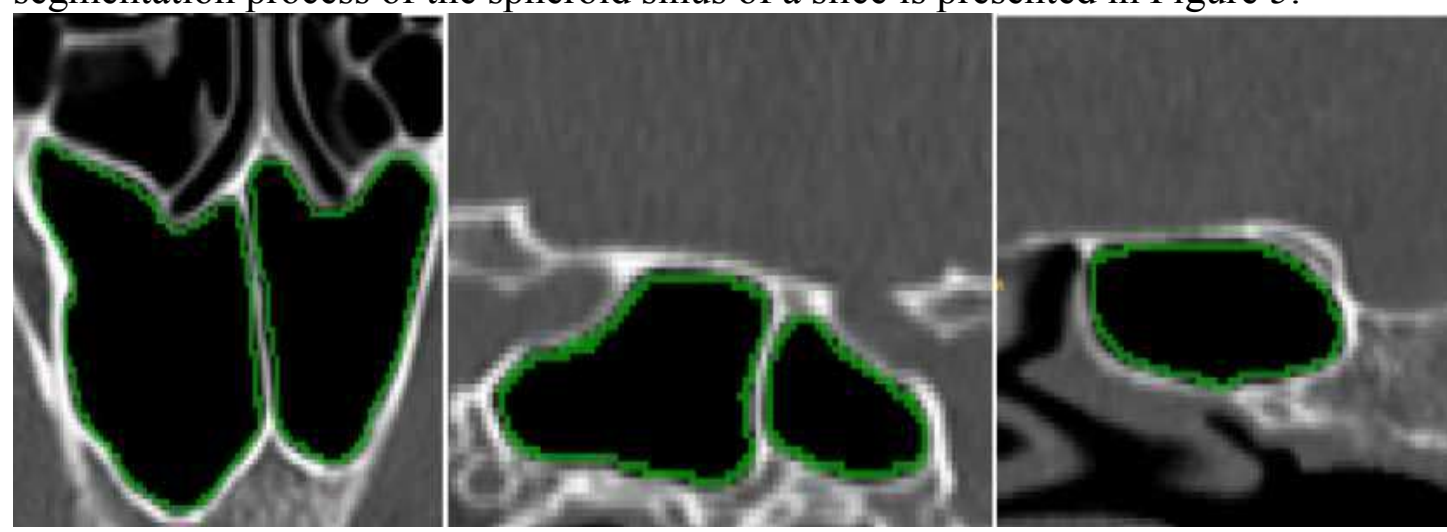

Fig. 5. Example of the process of manual segmentation on one slice. From left to right: an axial, sagittal and coronal view.

The DSC (Dice Similarity Coefficient, HD (Hausdorff distance ), and MAD (Mean Absolute Distance), were used for evaluating the proposed method. The dice Coefficient (DSC), one of the most commonly way for evaluating segmentation results, indicates a level of similarity between the reference (manual segmentation) and segmented result (automatic segmentation), the formulation of DSC is given by:

$$
D S C=\frac{2 N\left(S_{1} \cap S_{2}\right)}{N\left(S_{1}\right)+N\left(S_{2}\right)},(7)
$$

where $S_{1}$ and $S_{2}$ represent the obtained segmentation and the ground truth respectively (manual segmentation), and $N$ defines the number of pixels. $D C S \in[0,1]$, so that the closer DCS value to 1 , is the better segmentation.

The $H D$ is metric that represents the spatial distance between two point sets, i.e., is the maximum distance between two point sets $C 1$ and $C 2$, from each point $a \in C 1$ to point $b \in$ $C 2$ and vice versa. $\mathrm{HD}$ is defined as follows:

$$
H D\left(C_{1}, C_{2}\right)=\max \left(h\left(C_{1}, C_{2}\right), h\left(C_{2}, C_{2}\right)\right) \text {. }
$$

The MAD metric is given as follows:

$$
\operatorname{MAD}\left(C_{1}, C_{2}\right)=\frac{1}{2}\left[\frac{1}{n} \sum_{i=1}^{n} d\left(a_{i}, c_{2}\right)+\frac{1}{m} \sum_{j=1}^{m} d\left(b_{j}, c_{1}\right)\right] \text {,(9) }
$$

where the distance between the point $\mathrm{a}_{\mathrm{i}}$, and the closest point, $b_{j}$ is given by

with $b_{j} \in C_{2}$.

$$
d\left(a_{i}, \mathrm{C} 2\right)=\min \left\|b_{j}-\mathrm{a}_{\mathrm{j}}\right\|, \quad(10)
$$

The three obtained metrics DSC, HD, and MAD for all segmentations appear in Tables1 along with a comparison between the proposed automatic segmentation and semiautomatic clustering of ITK-SNAP for the nine CT- images respectively with manual segmentation. 
Table 1. Detailed results of comparison between the proposed automatic and semiautomatic (ITK-SNAP) segmentation for 9 volumes, using respectively DSC, HD, and MAD distances.

\begin{tabular}{|l|l|l|l|l|l|l|l|l|l|}
\hline $\begin{array}{l}\text { CT Volumes } \\
\text { 123456789 Our } \\
\text { results }\end{array}$ & 96.10 & 95.59 & 92.10 & 95.71 & 96.84 & 95.91 & 95.48 & 96.87 & 96.52 \\
\hline ITK-SNAP results & 96.10 & 95.74 & 96.74 & 95.21 & 97.15 & 96.16 & 95.77 & 96.33 & 95.78 \\
\hline Our results & 4.09 & 7.02 & 6.62 & 2.71 & 0.96 & 2.08 & 1.58 & 1.85 & 2.22 \\
\hline ITK-SNAP results & 6.88 & 6.61 & 2.06 & 0.93 & 2.10 & 1.54 & 2.01 & 2.20 & 4.08 \\
\hline Our results & 10.43 & 15.43 & 19.46 & 13.32 & 3.79 & 8.07 & 4.76 & 5.15 & 8.39 \\
\hline ITK-SNAP results & 15.43 & 19.46 & 6.29 & 3.87 & 8.19 & 4.67 & 5.18 & 8.41 & 43.76 \\
\hline
\end{tabular}

\section{Discussion}

CT and Augmented Reality (AR) can improve the work of otorhinolaryngologists because these tools help to investigate target and risk structures [21]. AR can be smoothly incorporated into the operating workflow.

The sphenoid sinus segmentation is a specific interesting problem and using Deep Learning (DL) to solve it is novel [22, 23, 24].

The methodology first cogitates ROI removal, followed by the 3D CNN application and some preprocessing. The methodology employs a standard 3D CNN previously used for medical image segmentation and analysis called DeepMedic.

Using 3D CNNs help deal with handcrafting and uncertainties but may pose problems related to Content-Based Image Retrieval (CBIR) [23, 25]. There are already end-to-end DL solutions, e.g., V-net or Seg-Net [26, 27] related to the segmentation. Later, the authors may compare the proposed methods with other architectures as well as investigate the bias. The chosen dataset is small since 15 annotated images have been used for training and 9 images for testing. Hence, it is very uncertain whether better conclusions can be made using other ampler datasets.

There is no evaluation of different $\mathrm{CNN}$ architectural variants or different types of optimizers. To improve this scheme, the authors should think about what the community can learn from the sphenoid sinus segmentation problem such as

(i) what type of data are specially hard;

(ii) similarities between this problem and other medical imaging segmentation applications;

(iii) if there is a novel solution for that specific problem;

(iv) if this kind of networks can be trained faster;

(v) if the design can be trained with the same precision;

(vi) how to pick up the right amount of data; and

(vii) the model is trained in a very constrained setting, where imagery containing fractures and so on has been removed; hence, investigations on how to train the models with all the data instead of removing the samples with fractures must be taken.

\section{Conclusion}

This reading tackled studies dealing with automatic segmentation of the sphenoid sinus via 3D CNN. The present study is the first initiative that found a decent correlation between the manual and automated sphenoidal sinus volume estimation techniques.

The proposed automated extraction of the sphenoidal sinus volume based on CT exams gives robust and accurate results close to the manual method where the reported outcomes 
are preliminary and may set up a good baseline if appropriately compared to other methodologies. This project results suggest that this automated implementation could be applied in clinical practice. It does not entail substantial user knowledge, besides being reproducible and fast.

\section{Acknowledgments}

The authors would like to thank Rabeh Djabri.

\section{Conflict of interest statement}

The authors certify that there is no conflict of interest with any financial organization in the subject matter or materials discussed in this manuscript.

\section{Authors' biography}

K. SOUADIH received his Master degree in computer science from Abderrahmane MIRA University, Bejaia, Algeria in 2015. Currently, his research interests include images analysis and processing by using machine and deep learning techniques in medical imaging.

A. Belaid - Associate Professor at Abderrahmane MIRA University, Bejaia, Algeria and Group Head of Image Processing at Medical Computing Laboratory (LIMED) University of Abderrahmane MiraBejaïaAlgeria.

D. Ben Salem, MD, PhD, is Professor of Radiology at the University of Western Brittany (Brest, FR). He received his medical and doctoral degree from the University of Burgundy (Dijon, FR). He is Editor-in-Chief of the Journal of Neuroradiology and member of other editorials boards (Heliyon and the Journal of Forensic Radiology and Imaging).

\section{References}

[1]. Giacomini, G. Pavan, A.L.M. Altemani, J.M.C. Duarte, S.B. Fortaleza, C.M.C.B. Miranda, J.R. \& Pina, D.R. (2018) Computed tomography-based volumetric tool for standardized measurement of the maxillary sinus, PLoS ONE, 13(1): e0190770. doi:10.1371/journal.pone.0190770 https://doi.org/10.1371/journal.pone.0190770 PMid:29304130 PMCid:PMC5755892

[2]. Knisely, A. Holmes, T. Barham, H. Sacks, R.\& Harvey, R.(2016) Isolated sphe $\neg$ noid sinus opacification: A systematic review American Journal of Otolaryngology, Head and Neck Medicine and Surgerhttps://doi.org/10.1016/j.amjoto.2017.01.014 PMid:28129912

[3]. Stokovic, N. Trkulja, V. Dumic-Cule, V. Cukovic-Bagic, I. T.L.S Vukicevic, T.L.S. \& Grgurevic, L. (2015) Sphenoid sinus types, dimensions and relationship with surrounding structures Annals of Anatomy (2015), doi: 10.1016/j.aanat.2015.02.013 PMid:25843780

[4]. Burke M.C., Taheri, R, Bhojwani, R \& Singh, A (2015) A Practical Approach to the Imaging Interpretation of Sphenoid Sinus Pathology Current Problems in Diagnostic, http://dx.doi.org/10.10677j.cpradiol.2015.02.002

[5]. Hacl, A. Costa, A.L.F. Oliveira, J.M. Tucunduva, M.J. Girondi, J.R, Raphaelli, A.C. \& Scocate, N. (2016) Three-dimensional volumetric analysis of frontal sinus using medical software. Journal of Forensic Radiology and Imaging https://doi.org/10.1016/j.jofri.2017.08.004

[6]. Wu, H.B. Zhu, L. Yuan, H.S. \& Hou, C. (2011) Surgical measurement to sphe $\neg$ noid sinus for the Chinese in Asia based on CT using sagittal reconstruction images, Eur Arch 


\section{Otorhinolaryngol (2011) $268 \quad$ :2)1 $\quad$ 2)6. $\quad$ https://doi.org/10.1007/s00405-010-1373-1} PMid:20857131

[7]. Guldnerc, C. Pistorius, S. Diogo, I. Bien, S. Sesterhenn, A.\& Werner, J. (2012) Analysis of pneumatization and neurovascular structures of the sphenoid sinus using cone-beam tomography (cbt)Acta. Radiol., vol. 53, no. 2, pp. 214-9, 2012 https://doi.org/10.1258/ar.2011.110381 PMid:22383784

[8]. Auffret, M. Garetier, M. Diallo I. Aho, S. \& Ben Salem, D. (2016) Contribution of the computed tomography of the anatomical aspects of the sphenoid sinuses to forensic identification J. Neuroradiol., vol. 43, no. 6, pp. 404414, 2016 https://doi.org/10.1016/j.neurad.2016.03.007 PMid:27083691

[9]. Uthman, A.T. AL-Rawi, N.H. Al-Naaimi,A.S. Tawfeeq A.S. \& Suhail E.H. (2009)Evaluation of frontal sinus and skull measurements using spiral CT scan $\neg n i n g$ : An aid in unknown person identification, Forensic Science International 197 (2010) 124.e1 124.e7 https://doi.org/10.1016/j.forsciint.2009.12.064 PMid:20097024

[10]. Kawari, Y. Fukushima, K. Ogawa,T. Nishizaki,K. Gunduz,M. Fujimoto, M.\& Yu Masuda (1999)Volume Quantification of Healthy Paranasal Cavity by Three-Dimensional CT Imaging $\begin{array}{llllll}\text { Acta Otolaryngol } & \text { (Stockh) 1999; } & \text { Suppl 540: } & 45 \neg 49\end{array}$ https://doi.org/10.1080/00016489950181198

[11]. Ahirwar, A. (2013) Study of Techniques used for Medical Image Segmen $\neg$ tation and Computation of Statistical Test for Region Classification of Brain MRII.J. Information Technology and Computer Science, 2013, 05, 4453 https://doi.org/10.5815/ijitcs.2013.05.06 [12]. Shen, D. Wu, G. \& Suk, H.(2017) Annu. Rev. Biomed. Eng. 2017. 19:22148 (The Annual Review of Biomedical Engineering is online at bioeng.annualreviews.org) https://doi.org/10.1146/annurev-bioeng-071516-044442 PMid:28301734 PMCid:PMC5479722

[13]. Srinivas, S. Sarvadevabhatla, R.K. Mopuri, K.R. Prabhu, N. Kruthiventi, S.S.S. \& Babu R.V. (2017) Introduction to Deep Convolutional Neural Nets for Com $\neg$ puter Vision Deep Learning for Medical Image Analysis -2017, Pages 25-52 https://doi.org/10.1016/B978-0-12810408-8.00003-1

[14]. Kamnitsas, K. Ledig, C. Newcombe, V.F.J. Simpson, J.P. Kane, A.D. Menon, D.K. Rueckert, D. \& Glocker, B. (2015) Efficient multi-scale 3D CNN with fully connected crf for accurate brain lesion segmentationproceeding of ISLES challenge, MICCAI 2015

[15]. Jani, A. Savsani, V. \& Pandya A. (2017) 3D Affine Registration using Teaching Learning Based Optimization, 3D Research Center, Kwangwoon Univer sity and Springer 2013https://doi.org/10.1007/3DRes.03(2013)2

[16]. Kamnitsas, K. Ferrante, E. Parisot, S. Ledig, C. Nori, A. Criminisi, A Rueckert, D. \& Glocker, B. (2016) DeepMedic for Brain Tumor Segmentation Biomedical Image Analysis Group https://doi.org/10.1007/978-3-319-55524-9_14

[17]. Simonyan, K. \& Zisserman, A. (2014) Very deep convolutional networks for large-scale image recognition, arXiv preprint arXiv:1409.1556 (2014)

[18]. Prionas, ND. Ray, S. \& Boone, JM. (2011) Volume assessment accuracy in computed tomography: a phantom study J Appl Clin Med Phys 2011; 11(2):3037. https://doi.org/10.1120/jacmp.v11i2.3037 PMCid:PMC5719953

[19]. Wafa, B., \& A. Moussaoui. A review on methods to estimate a CT from MRI data in the context of MRI-alone RT. Medical Technologies Journal, Vol. 2, no. 1, Mar. 2018, pp. 150178. https://doi.org/10.26415/2572-004X-vol2iss1p150-178

[20]. Rachida, Z., A. Belaid, \& Salem DB. A segmentation method of skin MRI 3D high resolution in vivo. Medical Technologies Journal, Vol. 2, no. 3, Sept. 2018, pp. 255-61, https://doi.org/10.26415/2572-004X-vol2iss3p255-261

[21]. Carl B, Bop M, Voellger B, Saß B, Nimsky C (2019) Augmented reality in transsphenoidal surgery, World Neurosurgery. doi: 10.1016/j.wneu.2019.01.202. https://doi.org/10.1016/j.wneu.2019.01.202 PMid:30763743

[22]. Razmjooy N, Estrela VV, Loschi H. J., Fanfan W. (2019) A comprehensive survey of new meta-heuristic algorithms, Recent Advances in Hybrid Metaheuristics for Data Clustering, Wiley Publishing. 
[23]. Hemanth, D.H. \& Estrela V.V. (2017) Deep Learning for Image Processing Applications, IOS. ISBN: 978-1-61499-821-1 (print) | 978-1-61499-822-8 (online)

[24]. Razmjooy, N., \& Estrela, V. V. (2019) Applications of Image Processing and Soft Computing Systems in Agriculture (pp. 1-337). Hershey, PA: IGI Global. doi:10.4018/978-15225-8027-0 https://doi.org/10.4018/978-1-5225-8027-0

[25]. Estrela, V. V., \& Herrmann, A. E. (2016) Content-based image retrieval (CBIR) in remote clinical diagnosis and healthcare. In M. Cruz-Cunha, I. Miranda, R. Martinho, \& R. Rijo (Eds.), Encyclopedia of E-Health and Telemedicine (pp. 495-520). Hershey, PA: IGI https://doi.org/10.4018/978-1-4666-9978-6.ch039

[26]. Badrinarayanan, V., Kendall, A., \& Cipolla, R. (2016) SegNet: A deep convolutional encoder-decoder architecture for image segmentation. IEEE Transactions on Pattern Analysis and Machine Intelligence, 39, 2481-2495. https://doi.org/10.1109/TPAMI.2016.2644615 PMid:28060704

[27]. Milletari, F., Navab, N., \& Ahmadi, S. (2016) V-Net: Fully convolutional neural networks for volumetric medical image segmentation. 2016 Fourth International Conference on 3D Vision (3DV), 565-571. https://doi.org/10.1109/3DV.2016.79 